Sobre los determinantes de la sostenibilidad fiscal de los municipios en Colombia. El caso de Antioquia, 2008-2017

Jorge Barrientos-Marín, Juan Felipe Quintero-Vanegas y Manuela Mendoza-Jaramillo 
Lecturas de Economía, 92 (enero-junio 2020), pp. 173-200

Jorge Barrientos-Marin, Juan Felipe Quintero-Vanegas y Manuela Mendoza-Jaramillo

Sobre los determinantes de la sostenibilidad fiscal de los municipios en Colombia. El caso de Antioquia, 2008-2017

Resumen: En este trabajo estamos interesados en establecer los determinantes de la sostenibilidad fiscal, entre ellas si las reglas de gasto, la denominada Ley 617 de 2000 coadyuvó a la promoción de la sostenibilidad de las finanzas públicas en los 125 municipios del departamento de Antioquia (Colombia) en el periodo 2008-2017. Para esto explotamos las heterogeneidades observables entre los 125 municipios de Antioquia en el período 2008-2017; debido a la endogeneidad de variables como deuda y el número de empresas usamos un estimador Hausman y Taylor. Nuestros resultados econométricos son estadísticamente significativos y los coeficientes estimados sugieren que la ley es efectiva en el aumento del ingreso tributario y el gasto de inversión, pero no son concluyentes sobre la efectividad en la disminución del gasto en funcionamiento. Otras variables de características de municipio como el número de empresas y el endeudamiento preexistente son explicativas y predictores del comportamiento de los ingresos tributarios, el gasto en funcionamiento y el gasto de inversión.

Palabras clave: finanzas públicas; sostenibilidad; regla de gasto; efecto aleatorio; endogeneidad.

Clasificación JEL: H1, H7, H60, C33.

On the determinants of fiscal sustainability of municipalities in Colombia. The case of Antioquia, 2008-2017

Abstract: In this work we are interested in establishing the determinants of fiscal sustainability, including if the spending rules, such as Law 617 of 2000 contributed to the promotion of the sustainability of public finances in the 125 municipalities of the department of Antioquia (Colombia) in the period 2008-2017. For this we exploit the observable heterogeneities between the 125 municipalities of Antioquia in the period 2008-2017, due to the endogeneity of variables such as debt and the number of companies we use a Hausman and Taylor estimator. Our econometrics results are statistical significant and the estimated parameters suggest that the law is effective in increasing tax revenue and investment spending, but they are not conclusive about effectiveness in reducing operating expenses; other variables of municipality characteristics such as the number of companies and pre-existing indebtedness are explanatory and predictive of the behavior of tax revenues, operating expenses and investment expenditure.

Keywords: public finances, fiscal sustainability, budget deficit, deficit bias, random and fixed effect, endogeneity.

JEL Classification: $H 1, H 7, H 60, C 33$.

Les déterminants de la viabilité budgétaire des municipalités de Colombie: Le cas d'Antioquia, 2008-2017

Résumé: Dans ce travail, nous soubaitons établir les déterminants de la viabilité budgétaire pour les 125 villes du département d'Antioquia (Colombie), au cours de la période 2008-2017. Il s'agit de savoir si les normes de la dépense publique, loi 617 de 2000, ont contribué à la viabilité des finances publiques. Pour ce faire, nous exploitons les bétérogénéités observables mais raison de l'endogénéité de variables, telles que la dette et le nombre d'entreprises, nous utilisons un estimateur Hausman et Taylor. Les résultats économétriques sont statistiquement significatifs et les coefficients estimés suggèrent que la loi 617 de 2000, est efficace en ce qui concerne la hausse des recettes fiscales et les dépenses d'investissement. Néanmoins, les résultats ne sont pas concluants en ce qui concerne l'efficacité dans la diminution des dépenses de fonctionnement. Les variables de chaque ville telles que le nombre d'entreprises et l'endettement préexistant, expliquent et prédisent le comportement des recettes fiscales, des dépenses de fonctionnement et des dépenses d'investissement.

Mots clés: finances publiques; durabilité; règle de dépenses; effet aléatoire; endogénéité

Classification JEL: H1, H7, H60, C33. 


\title{
Sobre los determinantes de la sostenibilidad fiscal de los municipios en Colombia. El caso de Antioquia, 2008-2017
}

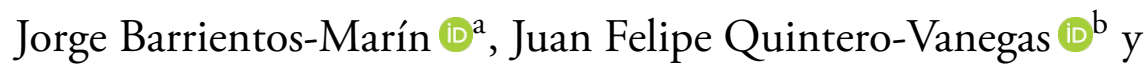 \\ Manuela Mendoza-Jaramillo
}

-Introducción. -I. Antecedentes. -II. Literatura relacionada. -III. Información estadística.

-IV. Estrategia empírica y resultados econométricos. -Conclusiones. -Anexos. -Referencias.

doi: 10.17533/udea.le.n92a06

Primera versión recibida el 22 de noviembre de 2019; versión final aceptada el 3 de diciembre de 2019

\section{Introducción}

La noción de insostenibilidad fiscal basada en el enfoque de endeudamiento y el balance fiscal puede ayudar a comprender hasta qué punto las reglas son eficientes y suficientes para que los países, departamentos y municipios adopten un comportamiento responsable frente a los recursos públicos, además de evaluar la capacidad de proveer bienes públicos esenciales a la población. Buena parte de la literatura existente sugiere que unas finanzas públicas sanas son importantes para explicar la promoción de la calidad de vida y el crecimiento económico. Contrasta con el hecho de que los gastos

* Este trabajo es derivado de un proyecto de investigación en el área de Descentralización Fiscal. Los autores agradecen la gestión del grupo GINVECO para llevarlo a cabo, cualquier error y mala interpretación es nuestra.

a Jorge Barrientos-Marin: profesor asociado de la Facultad de Ciencias Económicas de la Universidad Antioquia y profesor de cátedra de la Universidad Autónoma Latinoamericana.

Dirección electrónica: jorge.barrientos@udea.edu.co

https://orcid.org/0000-0001-5368-1928

b Juan Felipe Quintero-Vanegas: economista de la Universidad Autónoma Latinoamericana. Dirección postal: juan.quinterova@unaula.edu.co

https://orcid.org/0000-0001-9551-4108

c Manuela Mendoza-Jaramillo: economista de la Universidad Autónoma Latinoamericana. Dirección postal: manuela.mendozaja@unaula.edu.co

https://orcid.org/0000-0002-7584-4449 
puramente operativos (o de funcionamiento) muestran un efecto dual, de un lado muestran un efecto positivo en la calidad de vida, pero por otro lado un efecto negativo en el crecimiento económico, al ser un gasto improductivo (Quintero et al., 2016; Gyourku \& Tracy, 2002; Romero \& Strauch, 2008).

La experiencia de Colombia en políticas sobre reglas fiscales nace con la crisis fiscal en la década de 1990, durante este periodo el proceso de descentralización enfatizó principalmente en la generación de ingresos que en la estabilidad de los gastos, en aumentar la capacidad técnica y mejorar la habilidad de los municipios para manejar el presupuesto público. La crisis del UPAC encareció el tipo de interés, en consecuencia, muchos gobiernos locales se excedieron en su deuda lo que afectó la estabilidad macroeconómica del país. Como estrategia para solucionar la crisis se crearon las reglas de deuda — la ley 358 de 1997_ y la de gasto en funcionamiento - -la ley 617 del 2000_ . En entre otras medidas se creó el marco fiscal de mediano plazo y una ley que creó disposiciones para el pago del pasivo pensional. Bajo este contexto Chamorro y Urrea (2015) estiman un modelo de panel a nivel de municipios y gobernaciones para Colombia, donde evalúan el cumplimiento de la regla de gasto en funcionamiento y de deuda y su efecto en la sostenibilidad fiscal, en la especificación empírica los autores controlan por la existencia de superávit fiscales, la dependencia de transferencias del gobierno central, el tamaño pasivo pensional en relación a los ingresos totales y controlan también por características municipios. De las reglas y reformas fiscales sus resultados muestran que solo la regla de deuda ha sido efectiva en la disminución del saldo de deuda, por otra parte, se encuentra que la mayor dependencia de las transferencias es efectiva en el incremento del saldo de deuda.

Las reglas fiscales son efectivas tanto a nivel nacional como a nivel regional, este artículo busca contribuir sobre esta línea teórica, proveyendo evidencia empírica sobre el efecto de las reglas fiscales en las finanzas públicas locales, en particular si el cumplimiento de los umbrales estipulados en la Ley 617 de 2000, entre otras variables, ha sido efectiva. El enfoque de esta investigación no difiere por completo del utilizado por Quintero et al. (2016) y Bergman et al. (2016), quienes estudian la sostenibilidad fiscal desde la perspectiva de la deuda y el balance primario. 
Este artículo se delimita para los municipios del departamento de Antioquia, el segundo en importancia económica en Colombia en cuanto a su participación en el producto interno bruto. En la línea de los artículos anteriormente mencionados, hace uso de las reglas fiscales contenidas en la Ley 617 del 2000 (regla de gasto) y otras variables como determinantes de la sostenibilidad y su efecto sobre el ingreso tributario, funcionamiento y gasto de inversión.

Se espera que las reglas fiscales tengan efectos positivos en la inversión y negativo en el gasto en funcionamiento, esto es, evidenciar un efecto positivo sobre el crecimiento de la inversión y, al menos, estable en el gasto operativo como respuesta a las reformas de descentralización, lo que estaría respaldado por lo encontrado por Faguet (2008). Respecto al ingreso tributario se espera que el esfuerzo del cumplimiento de las reglas fiscales presione al aumento los ingresos tributarios, esta expectativa está sustentada por el análisis estadístico que arroja que los municipios con menores ingresos tributarios tienden, en promedio, a cumplir con la ley respecto aquellos con mejores ingresos

El incumplimiento conlleva sanciones en categorización de los municipios, generando un efecto dominó sobre los salarios de los funcionarios públicos y las transferencias de la nación de destinación específica y libre destinación; los municipios que incumplen la ley deben emprender un plan de recuperación fiscal que se presenta a la Secretaría de Planeación del departamento y a la Contraloría Departamental. Habida cuenta de lo anterior, es válido preguntarse el efecto que tuvo particularmente la Ley 617 en las finanzas públicas de los municipios del departamento de Antioquia para los años 2008-2017.

Para un enfoque local según Bergman et al. (2016) y Quintero et al. (2016), los desbalances fiscales están asociados a factores como la tragedia de los bienes comunes, la inconsistencia dinámica, y la visión de corto plazo de los gobernantes. En la investigación se controla por la deuda rezagada un periodo para el factor de inconsistencia dinámica; además se incluye una variable de elecciones que indica el año de gobierno desde la posesión hasta las elecciones; por otra parte, se utiliza variables de distancia desde Medellín para evaluar si existente spillovers que se expanden desde la ciudad de Medellín, como centro 
Barrientos-Marín, Quintero-Vanegas y Mendoza-Jaramillo: Sobre los determinantes...

económico, hacia los municipios del departamento de Antioquia según su lejanía o cercanía.

Este documento tiene cinco secciones además de esta introducción. La sección I presenta los antecedentes a las reglas fiscales y de gasto; la sección II se muestra literatura relacionada; en la sección III se expone la información estadística; la sección IV describe la metodología y resultados empíricos, y en la última sección se concluye.

\section{Antecedentes}

En la década de 1990 con las reformas estructurales en América Latina hubo una tendencia a la descentralización gubernamental. En Colombia por ejemplo se introdujo la elección de alcalde por voto popular en 1988 y, a su vez, aumentaron las transferencias del gobierno. Según Oates (1999), los gobiernos locales son más eficientes en la asignación de recursos públicos dado que tienen mejor información y más fuertes incentivos para hacer las cosas mejor que el gobierno nacional.

El manejo de las finanzas públicas afecta la inversión en bienes públicos, esto tiene lógica desde la teoría de crecimiento endógeno como lo indica Barro (1990), dado que el Estado promueve la productividad y el crecimiento económico desde distintas vías, como la provisión de capital fijo social, la promoción de la sinergia entre empresas, la fortaleza de las instituciones en materia de garantizar las libertades individuales, mejorar la infraestructura educativa y la generación de conocimiento e innovación. Es por esto que un limitado gasto en inversión puede afectar el crecimiento económico, tal como lo afirman Bergman et al. (2016), ya que las inadecuadas decisiones de los policy makers generan una disminución de la productividad total factorial. Es por ello importante entender cómo los limitantes de las reglas de deuda y gasto en funcionamiento afectan la sostenibilidad fiscal. El mismo Bergman et al. (2016) encuentra que las reglas fiscales, principalmente la ley de balance general es efectiva en la consecución de la sostenibilidad fiscal. 
En este punto es importante mencionar el delicado tema del estrés fiscal. Para Thompson (2017) y Gyourko y Tracy (2002), la clasificación de estrés fiscal tiene un efecto negativo en el precio de la vivienda, puesto que las variables fiscales son tomadas en cuenta por los agentes a la hora de comercializar bienes inmobiliarios, específicamente las viviendas. El estrés también produce recortes en el gasto de servicios públicos, seguridad e infraestructura. A su vez se encuentra que la inclusión de variables fiscales y servicios del gobierno tienen una importancia alta en relación con variables como la fortaleza de sindicatos públicos, el medio ambiente y las comodidades (bienes públicos).

\section{Literatura relacionada}

Desde la línea de política económica se han realizado diversos estudios para evaluar el efecto de la regla fiscal en la sostenibilidad. Bergman et al. (2016) hacen un análisis para la Unión Europea teniendo en cuenta factores como la fortaleza de la ley y su complementariedad con la eficiencia gubernamental en la promoción de la sostenibilidad y el balance fiscal. La fortaleza de las reglas fiscales se cuantifica con un indicador que incluye aspectos como el monitoreo, las bases legales y la cobertura, y utiliza las reglas de gasto en funcionamiento, los ingresos, el balance presupuestal y la deuda para su evaluación. La eficiencia gubernamental también juega un papel importante en la sostenibilidad, los autores utilizan un indicador del Banco Mundial que evalúa la percepción en la provisión de bienes públicos, la independencia de presiones políticas y la transparencia. Bergman et al. (2016) también encuentran que las reglas fiscales son efectivas en la promoción de la sostenibilidad fiscal y son complementarias a la eficiencia fiscal en un largo espectro de características.

Desde la literatura en política económica, el proceso de descentralización ha sido objeto de estudio, y uno de los enfoques corresponde a analizar cómo las reformas de descentralización de países como Colombia han afectado la inversión pública y el crecimiento económico. Para introducir los procesos de descentralización es importante entender los argumentos a favor y en contra 
Barrientos-Marín, Quintero-Vanegas y Mendoza-Jaramillo: Sobre los determinantes...

de la centralidad y el federalismo fiscal; para una amplia discusión al respecto véase Tiebout (1956) y Oates (1999).

La descentralización a mitades del siglo XX fue un proceso global, en Latinoamérica los casos de Colombia y Bolivia han sido los que más han suscitado interés entre los investigadores. En la literatura el proceso colombiano se describe como gradual con tres momentos claves. El primer momento es en la década de 1970, cuando se sustituye el impuesto a las ventas por el impuesto al valor agregado (IVA), es importante anotar que este impuesto pasa a ser el más representativo como porcentaje del recaudo total, en términos de descentralización se aumentan las transferencias de recursos por concepto de este impuesto alrededor del $50 \%$. El segundo momento se da a mediados de la década de 1980, cuando se hacen reformas que permiten la elección popular de los gobernadores y se crean las juntas administradoras locales para potenciar el desarrollo participativo y permitir la autogestión del gasto. El tercer momento ocurre en la década de 1990 con la reforma constitucional de 1991, la promulgación de las Leyes 715 y 60 que adjudican responsabilidades a los municipios en materia de planeación, financiación y provisión de bienes públicos y a su vez aumenta la intervención de los gobiernos locales en asuntos económicos y sociales (véase Faguet, 2008; Lozano y Julio, 2015; Chamorro y Urrea, 2016).

Para el periodo 1995-2010 las reformas arrojan un balance exitoso pues significó el aumento de la inversión total y el aumento de los ingresos totales. $\mathrm{Al}$ analizar los componentes del ingreso se encuentra que su crecimiento está explicado por las transferencias del gobierno en más de $50 \%$, el segundo rubro más importante son los ingresos tributarios que representó un incremento del $13 \%$. El comportamiento de los ingresos totales muestra que las reformas se centraron más en la descentralización del gasto que del ingreso como lo indican Faguet (2008) y Chamorro y Urrea (2016).

En la línea sobre calidad de vida y sostenibilidad fiscal Quintero et al. (2016) se centran en analizar la incidencia de un grupo de variables asociadas a las finanzas públicas usando un panel a nivel de municipios para las subregiones Oriente y Suroeste durante los años 2004-2014. Como variable proxy de calidad de vida se utiliza el indicador de necesidades básicas 
insatisfechas (NBI) y como vector de finanzas públicas se utilizan las variables de operaciones de caja de gobierno público (Ingresos y Gastos), deuda y superávit fiscal; por otra parte también se analiza la calidad de vida a nivel espacial para esto se realiza una georreferenciación y se incluyen dentro de la especificación del modelo general las variables dicotómicas de subregión (Oriente, Suroeste) y la variable distancia (Cerca o Lejos). Los resultados indican que la variable de finanzas públicas más efectiva en las condiciones de vida es el gasto en funcionamiento, por otra parte, la variable inversión muestra ser efectiva solo en el largo plazo. En cuanto a la georreferenciación se encontró que el pertenecer a la subregión oriente es efectivo y significativo en el mejoramiento de la calidad de vida, a su vez la cercanía con los centros económicos es importante en la calidad de vida lo que sugiere la existencia de efecto desbordamiento de las ciudades a los municipios.

La línea de investigación de política económica y sostenibilidad se centra en estrategias de política aplicadas empíricamente en gobiernos nacionales o locales para tratar problemas de insostenibilidad ante desbalances fiscales o niveles altos de endeudamiento. Desde un enfoque a nivel de país, Bergman et al. (2016), ya mencionado, realizan un estudio para 27 países de Europa que abarca el periodo 1990-2012, donde estudian cómo la fortaleza de las reglas fiscales y la eficiencia gubernamental aporta a la promoción de la sostenibilidad fiscal. Se incorporan en el estudio cuatro tipos de reglas fiscales: de gasto, de funcionamiento, de ingresos y de balance presupuestal. Se quiere comprobar si las variables "eficiencia fiscal" y "regla fiscal" aportan conjuntamente a la sostenibilidad de las finanzas públicas, es decir lo que comúnmente se conoce como la hipótesis de complementariedad. Los autores encuentran que las reglas fiscales están asociadas con políticas fiscales más sostenibles, este efecto se mantiene a través de un largo espectro de características de eficiencia gubernamental. En el análisis individual de reglas fiscales encuentran que no todas las reglas son efectivas en el mejoramiento de la sostenibilidad, la regla de ingresos es inefectiva mientras que la regla de balance presupuestal es la más efectiva de las cuatro reglas presentadas.

Desde un enfoque más local, Thompson (2017), estudia cómo el ser clasificado como un municipio con estrés fiscal afecta la sostenibilidad fiscal, para ello utiliza un modelo de panel para 932 municipios del estado de 
Barrientos-Marín, Quintero-Vanegas y Mendoza-Jaramillo: Sobre los determinantes...

Ohio en Estados Unidos en los años 1999-2012; se analiza en particular el efecto de recibir y de remover la clasificación de estrés fiscal ${ }^{1}$ en variables de operaciones de caja pública como ingresos y gastos, e inversión desagregada por sectores. Por otra parte, con el fin de hacer un análisis de cómo las malas decisiones fiscales afectan la calidad de vida vía eficiencia en la asignación de bienes públicos se hace un análisis alternativo del estrés fiscal utilizando precio de la vivienda y el crimen. Se encuentra que los gastos se reducen cerca de entre $23 \%$ y $25 \%$, luego de recibir la clasificación, la reducción de los gastos se da en gastos operativos, pero sobre todo en gastos de capital.

Como puede constatarse, los enfoques para el análisis de las políticas de sostenibilidad fiscal son distintos, autores como Bergman et al. (2016), se centran en la fortaleza de las reglas fiscales, mientras que Thompson (2017) se enfoca en las consecuencias de recibir la clasificación de estrés, quien señala, además, que tal clasificación tiene implícito el juicio de que las finanzas de un municipio son insostenibles, puesto que se prevé que esto afecta el gasto de inversión y por ende la inversión de bienes públicos. De otro lado Bergman et al. (2016) hacen un análisis directo de la eficiencia fiscal en la sostenibilidad fiscal, usando un indicador que mide la eficiencia fiscal desde la percepción de los ciudadanos en categorías como la provisión de bienes públicos, la inversión en obras civiles y la transparencia.

En la literatura la investigación que más se acerca a la nuestra es la de Chamorro y Urrea (2016), quienes evalúan los efectos de un paquete de políticas económicas implementadas en Colombia a finales de 1990 y principios del 2000 debido a una crisis fiscal por el sobreendeudamiento de los municipios. Las políticas corresponden a la Ley 617 del 2000 y la Ley 358 de 1997, como reglas fiscales, la primera es una regla de gasto y la segunda una regla de capacidad de pago de la deuda, donde se utiliza como variable de referencia la razón entre los intereses y el ahorro operacional, otras medidas son la Ley 549 de 1993 que contiene disposiciones sobre el pasivo pensional. Para la evaluación de los efectos de las políticas se hace un modelo de panel donde se analiza cómo el cumplimiento de las reglas fiscales, el superávit fiscal y la proporción de pasivo pensional en relación con los ingresos totales

1 La clasificación de estrés fiscal es asignada por el auditor del estado de Ohio, el recibir la clasificación exige a los municipios realizar planes de recuperación fiscal. 
afecta la sostenibilidad vista desde la deuda; también se consideran la variable dependencia económica para controlar como la relación entre la transferencia y los recursos propias afecta la deuda. Los autores encuentran que las medidas fiscales expuestas en la Ley 358 de 1997 es efectiva en la disminución del saldo de deuda, pero, por otra parte, encuentran que los municipios con mayor capacidad de generar recursos propios son más efectivos en la disminución del endeudamiento. Los resultados respaldan la investigación de Bergman et al. (2016) pero a nivel local, por lo que se puede inferir por la evidencia empírica de que la política de reglas fiscales es una herramienta efectiva en la promoción de la sostenibilidad fiscal.

\section{Información estadística}

La muestra utilizada en este trabajo está constituida por los 125 municipios del departamento de Antioquia para el periodo 2008-2017. La base de datos utilizada resulta de la concatenación de tres bases de datos diferentes: la del portal territorial del Departamento Nacional de Planeación (DNP), el DATLAS de Bancoldex y proyecciones de población del censo del Departamento Administrativo Nacional de Estadística (DANE) de 2005. Del portal territorial se obtiene información de finanzas públicas que contiene las operaciones de caja del gobierno e inversión por sectores registradas en el formato único tributario (FUT) y comprende las transferencias por el sistema general de participaciones (SGP). De esta base de datos se seleccionaron variables como ingresos tributarios locales, que se compone de impuesto predial, sobretasa a la gasolina, industria y comercio, la variable gasto en funcionamiento que hace parte de los gastos corrientes, que a su vez se compone de gastos en servicios personales, gastos generales y gastos por transferencias generadas por obligaciones del ejecutor y la variable gasto de capital que corresponde a las inversiones realizadas por la administración territorial en sectores de su competencia, como salud, educación, saneamiento, infraestructura, etcétera.

Adicionalmente, se calcula el ingreso corriente de libre destinación que corresponde a todos aquellos recursos tributarios y no tributarios que no son de asignación específica. Con este cálculo y utilizando la variable de gasto 
Barrientos-Marín, Quintero-Vanegas y Mendoza-Jaramillo: Sobre los determinantes...

en funcionamiento, se halla la proporción entre el gasto en funcionamiento y los ingresos corrientes de libre destinación. Es importante notar que la ley crea categorías municipales que segmentan cada municipio $i$ según los ingresos corrientes de libre destinación y el tamaño de la población, y a su vez crea una regla fiscal de gasto en funcionamiento, que corresponde a la razón entre el gasto en funcionamiento (GF) y los ingresos corrientes de libre destinación (ICLD) de los municipios. Dicha regla establece límites (umbrales) según categorías, por ejemplo, si el municipio $i$ está en categoría especial y $\frac{G F_{i}}{I C L D_{i}} \geq 0,5$ entonces tal municipio incumple la Ley 617. Para el resto de categorías los umbrales son los siguientes: primera categoría incumple si $\frac{G F_{i}}{I C L D_{i}} \geq 0,65$, segunda y tercera incumplen si $\frac{G F_{i}}{I C L D_{i}} \geq 0,70$ y cuarta, quinta y sexta incumplen si $\frac{G F_{i}}{I C L D_{i}} \geq 0,8$. Con esto en mente se crea la variable dicotómica "Ley617", que toma el valor de 1 cuando cumple la ley y el valor de 0 cuando no se cumple. Obviamente esta variable es variante temporal puesto que el incumplimiento de la regla del municipio ocasiona un cambio en la categoría (o en caso de que el municipio se encuentre en la categoría más baja que corresponde a la categoría 6 , el incumplimiento cambiaría la figura de municipio a corregimiento).

El saldo de deuda se extrae de la página del DNP. Esta variable es construida y es reportada por las cuentas FUT al DNP, contraloría general de la república y súper financiera. La base tiene como periodicidad los años 2000-2013 y esta se proyecta hasta el 2017 utilizando un método de suavizamiento a la Holt-Winters no estacional, para el vector de características de los municipios se emplean las variables empleo, empresas y población. Del DATLAS se obtienen las variables empresas y empleo.

Para el periodo analizado se evidencia una mayor frecuencia del cumplimiento con respecto al incumplimiento. El $83 \%$ de los municipios cumplen la Ley mientras el 17\% la incumplen. Al analizar la frecuencia de cumplimiento de forma dinámica se observa que hay una tendencia a la baja en el incumplimiento con ligeras fluctuaciones al alza en los años 2007, 2011 y 2015; como comparativo en el año 2003 cerca del $40 \%$ de los municipios incumplían la ley, para el 2017 el incumplimiento es marginal representando menos del $5 \%$. Si se discrimina el incumplimiento por categoría se encuentra 
que los municipios de categoría 5 y 6 presentan grandes mejorías con respecto a los municipios de las categorías restantes. La Figura 1 muestra la frecuencia de cumplimiento e incumplimiento de los municipios del departamento de Antioquia. Las Tablas 1 y 2 muestran las estadísticas descriptivas de las principales variables para ambos tipos de municipio.

Figura 1. Comportamiento del cumplimiento de la Ley 617

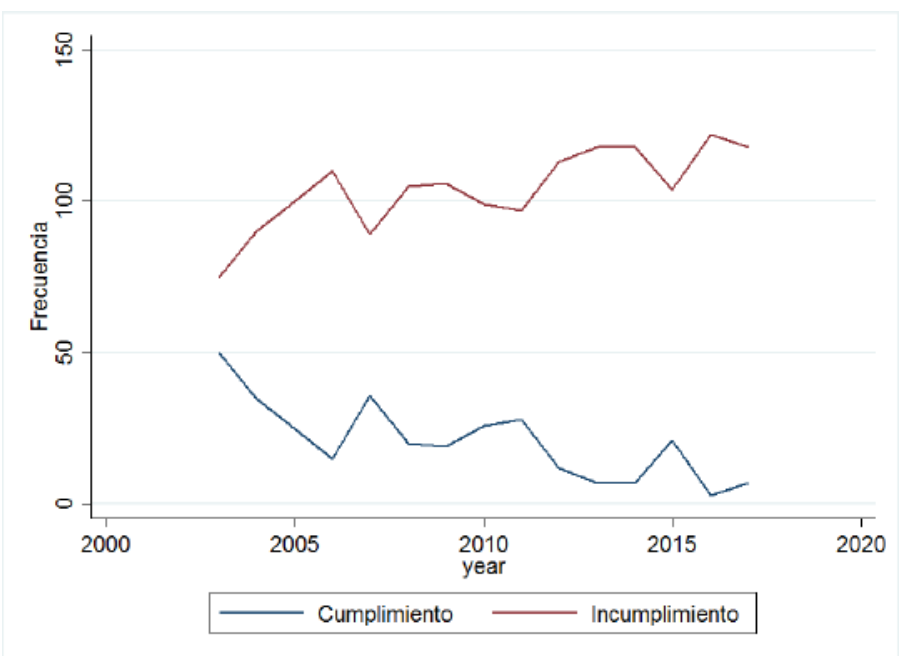

Fuente: elaboración propia con datos obtenidos de TERRIDATA, DNP.

La variable población corresponde a las proyecciones del CENSO del 2005, se encuentran en la sección demografía y población de su página Web, la variable elecciones es una variable categórica de 1 a 4 y que corresponde al año de gobierno en que se encuentra el alcalde, donde 1 es el año de posesión y 4 el año de finalización. La variable categorías es una clasificación que hace la Contaduría General de la nación según la ley 617 del 2000, ley que establece las categorías de los municipios según los ingresos corrientes de libre destinación, la población y el cumplimiento de los límites de gasto en funcionamiento, las categorías son una variable de selección múltiple donde la más alta categoría se llama especial, y sucesivamente continúa la categoría 1 hasta la categoría 6. Las variables están expresadas en pesos constantes. 
Barrientos-Marín, Quintero-Vanegas y Mendoza-Jaramillo: Sobre los determinantes...

Tabla 1. Municipios que no cumplen la Ley 617

\begin{tabular}{lccccc}
\hline & Media & Desviación estándar & Mínimo & Máximo & N \\
\hline Ingreso tributario & $70.577,16$ & $77.695,14$ & $1.184,581$ & $411.441,6$ & \\
Funcionamiento & $49.133,43$ & $34.918,35$ & 0 & $209.986,1$ & \\
Gasto de capital & $235.677,1$ & $175.949,8$ & $25.123,96$ & 1.264 .089 & 150 \\
Población & $39.796,01$ & $59.874,23$ & 2.128 & 413.186 \\
Saldo deuda & 0,6429527 & 3,003195 & - & 24,5673 \\
& & & 0,073012 & \\
Empleo & 854,4733 & $1.666,901$ & 0 & 7.196 \\
Empresas & 52,80667 & 89,58755 & 0 & 493 \\
\hline
\end{tabular}

Nota: la información de las operaciones de caja del gobierno público está expresada en pesos per cápita. Las cifras están en millones de pesos.

Fuente: elaboración propia con datos terridata, 2017.

El mayor número de empresas se concentra en los municipios de categoría especial, luego en magnitud le sigue los municipios de la categoría 6, este resultado se debe a que el $84 \%$ de los municipios de Antioquia para el 2018 pertenecen a dicha categoría. Al hacer el análisis temporal se identifica una tendencia al alza para la categoría especial y primera. Es de notar que desde 2010 para la categoría primera se ha dado una tasa de crecimiento elevada, esta tendencia puede obedecer al proceso de transformación productiva que vive la ciudad Medellín, cambiando su vocación de una ciudad industrial a una ciudad de servicios, lo que ha originado un desplazamiento geográfico de las empresas de Medellín hacia municipios como Barbosa, Copacabana, en el norte del área metropolitana y municipios como Envigado, Itagüí y Caldas, los cuales se encuentran ubicados en el sur del área metropolitana.

También hay una diáspora de empresas de Medellín hacia el oriente Antioqueño, en la zona del valle de San Nicolás, donde se destacan los municipios de Rionegro y Guarne. Se encuentra que la densidad empresarial es mayor en los municipios aledaños al área metropolitana, lo que se explica por la movilidad de factores como capital, capital humano y trabajo, la sinergia 
y el relacionamiento con el sector productivo, los efectos de desbordamiento de conocimiento debido a las actividades productivas de mayor complejidad y la existencia de economías del conocimiento. La Figura 2 muestra la distribución de la dinámica empresarial por categoría de municipio y por año.

Tabla 2. Municipios que cumplen la Ley 617

\begin{tabular}{lccccc}
\hline & Media & Desviación Estándar & Mínimo & Máximo & N \\
\hline Ingresos tributarios & $53.009,52$ & $59.066,6$ & 0 & $553.744,3$ & \\
Funcionamiento & $48.927,15$ & $35.907,2$ & 0 & $317.468,2$ & \\
Gasto de capital & $260.300,5$ & $172.669,8$ & 0 & 1.706 .837 & \\
Población & $51.384,64$ & $231.120,7$ & 2019 & 2.508 .452 & \\
Saldo deuda & 0,8965813 & 7,685751 & $-0,5601$ & 160,5543 & 1.100 \\
Empleo & $1.254,905$ & $8.170,496$ & 0 & 110.104 & \\
Empresas & 64,39727 & 352,5363 & 0 & 4.667 & \\
\hline
\end{tabular}

Nota: la información de las operaciones de caja del gobierno público está expresada en pesos per cápita. Las cifras están en millones de pesos.

Fuente: elaboración propia con datos de TERRIDATA, 2017.

Para los municipios de categoría 6 se evidencia un estancamiento en la densidad empresarial, esto se puede relacionar con la dinámica de cumplimiento dado que el comportamiento en la creación de empresas formales afecta los ingresos tributarios debido a que una de las fuentes de dichos ingresos es impuesto de cámara y comercio; si los municipios de esta categoría cuentan con poca capacidad de generación de ingresos propios y dependen de transferencias, hay mayor probabilidad de incumplir la regla de gasto; es por esto que sorprende el comportamiento de la frecuencia de cumplimiento, puesto que es probable que los planes de recuperación fiscal causen grandes esfuerzos a los municipios para disminuir los recursos destinados a gasto en funcionamiento en relación al gasto de inversión.

En términos per cápita, para los municipios que cumplen la ley en relación con los que no la cumplen, el gasto en funcionamiento es menor marginalmente y la inversión es mayor en tres mil pesos. Por otra parte, se 
Barrientos-Marín, Quintero-Vanegas y Mendoza-Jaramillo: Sobre los determinantes...

encuentra que los ingresos tributarios son menores por 17 mil pesos y el endeudamiento es mayor, corresponde a 0,89 pesos para los municipios que cumplen con respecto a 0,64 pesos para los que incumplen. Se puede afirmar que hay mayor sostenibilidad vista desde una mayor proporción de recursos invertidos en gasto en inversión con respecto a gasto en funcionamiento, pero hay insostenibilidad vista desde el mayor endeudamiento y los menores ingresos tributarios.

Figura 2. Dinámica empresarial por categorías

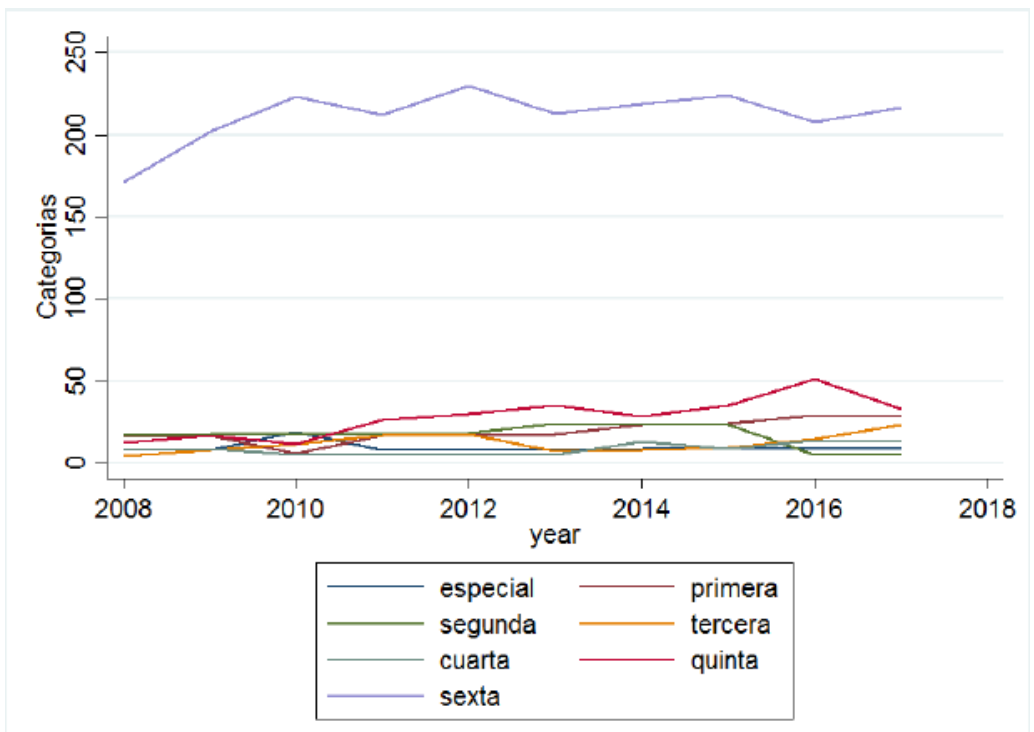

Fuente: elaboración propia con datos obtenidos de la Contaduría General de la Nación y BANCOLDEX, 2017.

Como resultado preponderante se obtiene que los municipios que cumplen la ley atraen y generan más empresas, en promedio hay 64 empresas formales con respecto a 42 para los municipios que cumplen. Los municipios que cumplen los umbrales tienen en promedio 51.384 habitantes mientras los que incumplen tienen 39.797, esto puede corresponder al hecho que los municipios que invierten menos bienes públicos per cápita incentivan a la población a emigrar a otros municipios, lo que es consistente el resultado de 
empresas y de la variable población con el de empleados formales, dado que los municipios que cumplen la ley cuentan con 1.254 empleados en promedio con respecto a 854 para los no cumplen. La mayor población, número de empleados y empresas sugiere que los municipios que cumplen tienen mayor crecimiento y desarrollo desde la eficiencia que los que no cumplen.

\section{Estrategia empírica y resultados econométricos}

\section{A. Modelos a estimar}

La estrategia de estimación en este artículo está basada en la siguiente especificación empírica:

$$
y_{i t}=x_{i t}^{\prime} \beta+u_{i t} \quad i=1, \ldots, n ; \quad t=1, \ldots, T,
$$

donde $u_{i t}=\gamma_{i}+v_{i t}, \gamma_{i}$ es una heterogeneidad no observable y $v_{i t}$ error clásico tal que $E\left(v_{i t} \mid x_{i t}\right)=0$ y $E\left(v_{i t}^{2} \mid x_{i t}\right)=\sigma_{v}^{2} \forall i t$. Entiéndase por idiosincrático aquellas características culturales, tradiciones, creencias y demás características del individuo o grupo específico que son no observables. La heterogeneidad $\gamma_{i}$ puede ser fija o aleatoria, si es fija entonces es un parámetro por estimar y si es aleatorio induce una matriz de varianzas y covarianzas con autocorrelación inducida y de forma conocida.

En este modelo $y_{i t}$ es un vector de variables proxy de sostenibilidad fiscal, para tres especificaciones de la regresión: (1) el ingreso tributario, (2) el gasto en funcionamiento y (3) gasto de capital. Las variables explicativas en la matriz $x_{i t} i=1, \ldots, n ; t=1, \ldots, T$ contiene variables como la variable Ley 617 , la cual se define como una variable binaria $=1$ cuando el municipio $i$ cumple la ley en el momento $t$ y es $=0$ en otro caso, el número de empresas, el número de empleados, el saldo de deuda en el periodo anterior dado que la deuda es una variable que tiene un efecto rezagado en la sostenibilidad fiscal y la población; contiene también la variable Categoría del municipio en el momento $t$ y la variable elecciones es una variable binaria que toma el valor $=1$ en el año en que hubo elecciones e $=0$ en otro caso. Finalmente, la variable distancia que mide el efecto de la cercanía a los centros de aglomeración económica en la sostenibilidad de los municipios. 
Barrientos-Marín, Quintero-Vanegas y Mendoza-Jaramillo: Sobre los determinantes...

En la teoría econométrica a parte de los criterios ya mencionados para la selección entre el modelo de efectos fijos y aleatorios, se utiliza la prueba de Hausman (1978), que indica la hipótesis nula del modelo es la exogeneidad del componente de error del grupo específico con los regresores del modelo. En caso de rechazar Ho, como es previsible, se usará un estimador de Hausman y Taylor (1981) que tiene la siguiente especificación:

$$
y_{i t}=x_{i t}^{\prime} \beta+z_{i}^{\prime} \phi+u_{i t} \quad i=1, \ldots, n ; \quad t=1, \ldots, T .
$$

En este modelo $x_{i t}$, corresponde al vector de variables variantes en el tiempo y $z_{i}$ a las variables invariante-temporales. Cada vector de variables se expresan de la siguiente forma $x_{i t}=\left[x_{1}, x_{2}\right] z_{i}=\left[z_{1}, z_{2}\right]$ donde $x_{1}$ es $n * k_{1}$; $x_{2}$ es $n * k_{2} ; z_{1}$ es $n * g_{1} ; z_{2}$ es $n * g_{2}$. Las matrices $x_{1}$ y $z_{1}$ son exógenas. Se utiliza el método mínimo cuadrados en dos etapas (2SLS) usando como instrumento las variables exógenas, así como las medias y desviaciones de la media de las variables exógenas para completar el rango de la matriz de instrumentos.

\section{B. Resultados empiricos}

La muestra utilizada es un panel con 125 observaciones de corte transversal y 9 observaciones de corte longitudinal, durante el periodo 20082017. En esta sección reportamos en las Tablas 3, 4 y la Tabla 1A (en el Anexo) los resultados para las estimaciones de los modelos especificados en las ecuaciones (1) y (2), para el estimador de efecto aleatorio, el de variables instrumentales y el de efecto fijo respectivamente. La prueba de Hausman y Taylor (1978) de exogeneidad de los regresores rechaza la hipótesis nula al $1 \%$ y $5 \%$ de significancia, lo cual indica que debe usarse instrumentos para resolver el problema de endogeneidad. Claramente la metodología requiere identificar qué variables son endógenas, en este trabajo son el número de empresas y la deuda. De un lado, entre los no observables que puedan causar la presencia de endogeneidad al estar correlacionadas con el número de empresas se consideran, por ejemplo: bienes públicos para el emprendimiento, como por ejemplo las exenciones tributarias, la creación de centros de aglomeración económica, y la creación de cooperativas que contribuyan a la generación de efectos de sinergia y faciliten el acceso a la financiación. 
Tabla 3. Modelo de efectos aleatorios

Determinantes del ingreso tributario (1), funcionamiento (2) y gasto de Inversión (3)

\begin{tabular}{|c|c|c|c|}
\hline & (1) & (2) & (3) \\
\hline \multirow[t]{2}{*}{ Ley617 } & $0,108^{*}$ & $0,0748^{*}$ & $0,128^{* *}$ \\
\hline & $(0,0474)$ & $(0,0377)$ & $(0,0427)$ \\
\hline \multirow{2}{*}{ Deuda $(t-1)$} & $0,0226^{*}$ & $0,0231^{* *}$ & $0,0252^{* *}$ \\
\hline & $(0,00973)$ & $(0,00781)$ & $(0,00887)$ \\
\hline \multirow[t]{2}{*}{ Población } & $0,514^{* * *}$ & $0,321^{* * *}$ & $0,459^{* * *}$ \\
\hline & $(0,0642)$ & $(0,0411)$ & $(0,0470)$ \\
\hline \multirow[t]{2}{*}{ Empresas } & $0,329^{* * *}$ & $0,186^{* * *}$ & $0,172^{* * *}$ \\
\hline & $(0,0384)$ & $(0,0287)$ & $(0,0327)$ \\
\hline \multirow[t]{2}{*}{ Empleo } & $0,0557^{*}$ & 0,0323 & 0,00806 \\
\hline & $(0,0238)$ & $(0,0179)$ & $(0,0204)$ \\
\hline \multirow[t]{2}{*}{ Categoría 2} & $-0,249$ & $-0,423^{* *}$ & $-0,585^{* * *}$ \\
\hline & $(0,195)$ & $(0,142)$ & $(0,162)$ \\
\hline \multirow[t]{2}{*}{ Categoría 3} & $-0,325$ & $-0,604^{* * *}$ & $-0,540^{* *}$ \\
\hline & $(0,211)$ & $(0,153)$ & $(0,174)$ \\
\hline \multirow[t]{2}{*}{ Categoría 4} & $-0,692^{* *}$ & $-0,985^{* * *}$ & $-1,016^{* * *}$ \\
\hline & $(0,240)$ & $(0,172)$ & $(0,196)$ \\
\hline \multirow[t]{2}{*}{ Categoría 5} & $-0,756^{* *}$ & $-1,042^{* * *}$ & $-0,913^{* * *}$ \\
\hline & $(0,233)$ & $(0,158)$ & $(0,181)$ \\
\hline \multirow[t]{2}{*}{ Categoría 6} & $-1,128^{* * *}$ & $-1,303^{* * *}$ & $-1,150^{* * *}$ \\
\hline & $(0,235)$ & $(0,158)$ & $(0,181)$ \\
\hline \multirow[t]{2}{*}{ Categoría Especial } & 0,124 & $0,615^{* *}$ & $0,653^{* *}$ \\
\hline & $(0,289)$ & $(0,210)$ & $(0,239)$ \\
\hline \multirow[t]{2}{*}{ Elecciones 2} & $0,760^{* * *}$ & $0,712^{* * *}$ & $0,865^{* * *}$ \\
\hline & $(0,0340)$ & $(0,0275)$ & $(0,0312)$ \\
\hline
\end{tabular}


Barrientos-Marín, Quintero-Vanegas y Mendoza-Jaramillo: Sobre los determinantes...

Tabla 3. Continuación

\begin{tabular}{lccc}
\hline & $(1)$ & $(2)$ & $(3)$ \\
\hline Elecciones 3 & $0,366^{* * *}$ & $0,316^{* * *}$ & $0,520^{* * *}$ \\
& $(0,0381)$ & $(0,0308)$ & $(0,0350)$ \\
Elecciones 4 & $0,100^{*}$ & 0,000885 & $0,265^{* * *}$ \\
& $(0,0389)$ & $(0,0314)$ & $(0,0356)$ \\
Distancia & $-0,00100^{*}$ & $-0,0000232$ & $0,0126^{* * *}$ \\
& $(0,000504)$ & $(0,000298)$ & $(0,000342)$ \\
Constante & $14,77^{* * *}$ & $17,31^{* * *}$ & $17,29^{* * *}$ \\
& $(0,711)$ & $(0,457)$ & $(0,523)$ \\
\hline$N$ & 1.125 & 1.124 & 1.124 \\
$R^{2}$ & 0,8224 & 0,8330 & 0,7966 \\
\hline
\end{tabular}

Nota: ${ }^{* *},{ }^{* *},{ }^{*}$ indican niveles de significancia al $1 \%, 5 \%, 10 \%$, respectivamente. Los errores estándar están entre paréntesis.

En la línea teórica de economía espacial, las decisiones de localización de una empresa pueden verse influenciadas por economías de localización, estas economías atraen a las empresas que buscan que el proceso de acumulación de capital exhiba rendimiento a escala, entre los factores encontramos encadenamientos productivos, oferta de bienes intermedios y primarios, relacionamiento con la cadena de suministro, competencia, capacidad de innovación, aprovechamiento de las potencialidades productivas del territorio y del capital humano. Estas variables influyen sobre la asignación de bienes públicos para el desarrollo productivo, lo que afecta el gasto en funcionamiento y gasto de inversión, a su vez son factores claves en el desarrollo y crecimiento de la economía de los gobiernos locales, afectando tanto los ingresos como los gastos, según Camagni (2015).

De otro lado, la deuda es endógena debido a que el gobierno central ante un escenario de crisis o recesión tiene herramientas de política fiscal para hacer política contra cíclica, una política fiscal expansiva desde la demanda agregada genera planes de expansión cuantitativa del gasto, estos planes impactan en los ingresos, gastos y endeudamiento; otro factor no observable que puede 
causar presencia de endogeneidad en la variable deuda son los choques de tipo externo como por ejemplo el comportamiento de los socios comerciales y la disminución de los términos de intercambio; ambos choques afectan los ingresos y gastos del gobierno nacional central y los gobiernos locales.

Se evidencia que la ley es efectiva en el incremento del gasto de inversión, el estimador del efecto del cumplimiento es significativo al $5 \%$ y tiene un efecto positivo equivalente al $0,128 \%$; el modelo con gasto en funcionamiento es significativo al 10\%, el efecto del cumplimiento es de $0,074 \%$, este efecto es positivo, contrario a lo esperado en la hipótesis, aunque es notable que el estimador de gasto en funcionamiento es menor al estimador de gasto de inversión, lo que sugiere mayor sostenibilidad en las finanzas públicas, por otra parte el cumplimiento de la ley mejora el ingreso tributario en $0,10 \%$ y es significativo al $10 \%$. La ley 617 también incentiva una mejora en los ingresos tributarios de los municipios. La variable deuda es significativa para el gasto en funcionamiento y gasto en inversión al $5 \%$; un aumento en $1 \%$ en el saldo de deuda del año previo aumenta el gasto en funcionamiento en $0,023 \%$, aumenta el gasto en inversión en 0,025 , este resultado nos dice que la deuda se reparte por partes casi equivalentes para el gasto en funcionamiento e inversión, por lo que no necesariamente la deuda local está sustentada en deuda productiva que permita la provisión de bienes públicos.

Las variables población y empresas explican a un nivel de significancia menor al $1 \%$ la sostenibilidad de las finanzas públicas. Este resultado se debe en parte a que el recaudo de impuestos locales como predial e industria y comercio dependen de estas variables, el efecto de la población en el gasto de inversión es mayor al gasto en funcionamiento, esto se puede interpretar desde la hipótesis de Tiebout (1961), dado que la población manifiesta sus preferencias desplazándose a los municipios que provean bienes públicos más eficientes y que se acerquen a las preferencias de los ciudadanos. Para el ciclo político el encontrarse en el segundo año de gobierno aumenta los ingresos tributarios en $0,76 \%$, este efecto disminuye paulatinamente, lo que sugiere que hay mayor probabilidad de un aumento en la tasa impositiva para personas naturales y jurídicas en los primeros dos años de gobierno. Con respecto al gasto en funcionamiento e inversión tienen un comportamiento 
Barrientos-Marín, Quintero-Vanegas y Mendoza-Jaramillo: Sobre los determinantes...

bastante similar al ingreso tributario, el mayor efecto se da en el año 2 y baja paulatinamente, en el gasto en funcionamiento la magnitud de los coeficientes para el año 3 y 4 es mucho menor a los estimadores para los mismos años del gasto en inversión.

Tabla 4. Estimación por variables instrumentales (Hausman y Taylor, 1981)

Determinantes del gasto en funcionamiento (1) y gasto de Inversión (2)

\begin{tabular}{|c|c|c|}
\hline & (1) & (2) \\
\hline \multirow[t]{2}{*}{ Ley617 } & 0,0609 & $0,0808^{* *}$ \\
\hline & $(0,0370)$ & $(0,0412)$ \\
\hline \multirow[t]{2}{*}{ Población } & $0,312^{* * *}$ & $0,407^{* * *}$ \\
\hline & $(0,0632)$ & $(0,0981)$ \\
\hline \multirow[t]{2}{*}{ Empleo } & 0,0298 & 0,0287 \\
\hline & $(0,0194)$ & $(0,0218)$ \\
\hline \multirow[t]{2}{*}{ Categoría 2} & $-0,281$ & $-0,229$ \\
\hline & $(0,160)$ & $(0,187)$ \\
\hline \multirow[t]{2}{*}{ Categoría 3} & $-0,478^{* *}$ & $-0,173$ \\
\hline & $(0,176)$ & $(0,208)$ \\
\hline \multirow[t]{2}{*}{ Categoría 4} & $-0,813^{* * *}$ & $-0,647^{* *}$ \\
\hline & $(0,204)$ & $(0,243)$ \\
\hline \multirow[t]{2}{*}{ Categoría 5} & $-0,865^{* * *}$ & $-0,555^{*}$ \\
\hline & $(0,205)$ & $(0,251)$ \\
\hline \multirow[t]{2}{*}{ Categoría 6} & $-1,066^{* * *}$ & $-0,750^{* *}$ \\
\hline & $(0,209)$ & $(0,257)$ \\
\hline \multirow[t]{2}{*}{ Categoría Especial } & 0,375 & 0,225 \\
\hline & $(0,235)$ & $(0,275)$ \\
\hline \multirow[t]{2}{*}{ Elecciones 2} & $0,708^{* * *}$ & $0,859^{* * *}$ \\
\hline & $(0,0263)$ & $(0,0290)$ \\
\hline
\end{tabular}

Continúa 
Tabla 4. Continuación

\begin{tabular}{lcc}
\hline & $(1)$ & $(2)$ \\
\hline Elecciones 3 & $0,312^{* * *}$ & $0,511^{* * *}$ \\
Elecciones 4 & $(0,0296)$ & $(0,0326)$ \\
& $-0,00609$ & $0,246^{* * *}$ \\
Empresas & $(0,0302)$ & $(0,0333)$ \\
& $0,242^{* * *}$ & $0,291^{* * *}$ \\
Deuda $(t-1)$ & $(0,0323)$ & $(0,0359)$ \\
& $0,0224^{* *}$ & $0,0226^{* *}$ \\
Distancia & $(0,00757)$ & $(0,00836)$ \\
& 0,0000739 & 0,00157 \\
Constante & $(0,000493)$ & $(0,000913)$ \\
& $17,07^{* * *}$ & $17,07^{* * *}$ \\
$R^{2}$ & $(0,699)$ & $(1,046)$ \\
\hline
\end{tabular}

Nota: ${ }^{* * *},{ }^{* *},{ }^{*}$ indican niveles de significancia al $1 \%, 5 \%, 10 \%$, respectivamente. Los errores estándar están entre paréntesis.

Fuente: elaboración propia.

La distancia es estadísticamente significativa para el gasto de inversión, el aumentar $1 \mathrm{~km}$ la distancia se aumenta el gasto de inversión en 0,0126 \%, en la hipótesis se esperaba que el efecto fuera negativo dado que, a mayor cercanía con Medellín, el municipio con mayor complejidad económica (capacidad de generar valor agregado), la inversión debe de ser mayor debido a los "spillover" del conocimiento aún con el signo esperado el efecto es muy pequeño.

Al utilizar la prueba de Hausman para testear la hipótesis de no correlación entre el componente de error de grupos específicos del modelo de efectos aleatorios y los regresores del modelo se encuentra que no se rechaza $H_{0}$ para el modelo de ingreso tributario, por lo que se puede utilizar el modelo 
de efectos aleatorios. Para el modelo de gasto en funcionamiento y gasto en inversión se rechaza $H_{0}$ con un nivel de significancia menor al $1 \%$, por lo anterior se emplea el modelo Hausman y Taylor (1981), que posee estimadores consistentes y eficientes para las variables invariantes en el tiempo que sean endógenas. El modelo de Hausman y Taylor en su forma funcional exige que se haga segmentación de las variables independientes que se correlacionan con el componente de error individual del modelo de efectos aleatorios, a su vez se segmentan las variables variantes en el tiempo e invariantes en el tiempo.

Se evidencia que el hecho de cumplir la ley no es significativo en la variable gasto en funcionamiento, aunque con el signo esperado, adicionalmente el cumplir la ley incrementa el gasto de inversión en 0,08 y es significativo a menos del $5 \%$ de significancia; el resultado de la variable deuda continúa siendo positivo y significativo, lo mismo ocurre con la variable empleo y población, el año 2 es el año donde más se incrementa el gasto en funcionamiento y de capital. Con respecto al modelo de efectos aleatorios para el modelo de gasto en inversión la variable distancia pasa a ser no significativa estadísticamente. Esto significa que controlar por la indogeneidad da un resultado esperado, pues la ley desincentiva el gasto excesivo en funcionamiento.

\section{Conclusiones}

Este artículo presenta un estudio de panel para el departamento de Antioquia en los años 2008-2017, se centra los determinantes de la sostenibilidad fiscal, en particular en la regla de gasto en funcionamiento contenida en la Ley 617 del 2000, cuya evidencia empírica sugiere que su cumplimiento, afecta a las finanzas públicas de los gobiernos locales. Además, se incluyen otros regresores tales como: 1 ) deuda (medida en el periodo $t-1$ ) para examinar la inercia de la deuda y su influencia en el nivel contemporáneo de las variables dependientes, 2) número de empresas, 3) nivel de empleo, 4) tamaño de la población 5) distancia, como características de municipio y 6) una variable de elecciones para examinar el ciclo político y una variable para controlar por la categorización del municipio debido al umbral que 
establece la Ley 617. Como variable dependiente se utilizan las variables ingreso tributario, gasto en funcionamiento y gasto de inversión.

La evidencia sugiere que el cumplimiento de la ley contribuye al incremento de la inversión. La ley 617 muestra ser significativa para persuadir el exceso de gasto en funcionamiento, este hecho es consistente con las estadísticas que muestran que en promedio los municipios que cumplen el umbral poseen más inversión y menor gasto en funcionamiento. Por otra parte, el esfuerzo de los gobiernos locales por cumplir la ley incrementa los ingresos tributarios, esto se correlaciona con las estadísticas donde se muestra que los municipios que cumplen tienen menores ingresos tributarios per cápita por lo que deben de realizar un mayor esfuerzo fiscal para poder cumplir la ley, lo que podría crear los incentivos para incrementar el esquema tributario local.

La evolución de la regla en términos de frecuencia de cumplimiento ha visto una tendencia al alza durante el periodo de análisis, este crecimiento ha contribuido a que en el 2008 el $40 \%$ de municipios incumpliera y en el 2017 tan solo el 5\%. Por lo anterior se puede inferir que la regla de gasto en funcionamiento ha contribuido al mejoramiento de la sostenibilidad fiscal. Los resultados se relacionan con los hallazgos de Faguet (2008) que encuentra que el proceso de descentralización en Colombia condujo a aumentos en la inversión y mantuvo los costos operativos estables; a su vez es consistente con los resultados de Bergman et al. (2016) que muestran que la fortaleza de las reglas fiscales contribuye a la sostenibilidad fiscal.

Como agenda para el futuro, es importante evaluar el efecto de otro tipo de reglas fiscales distintas a la de gasto en funcionamiento, como reglas de ingresos, deuda o balance presupuestal y ver cómo estas reglas complementan a la regla de gasto en funcionamiento en el objetivo de mejorar la sostenibilidad fiscal de los municipios. 
Barrientos-Marín, Quintero-Vanegas y Mendoza-Jaramillo: Sobre los determinantes...

\section{Anexo}

Tabla 1A. Efectos fijos (estimador intragrupos)

Determinantes del ingreso tributario (1), funcionamiento (2) y gasto de Inversión (3)

\begin{tabular}{|c|c|c|c|}
\hline & (1) & (2) & (3) \\
\hline \multirow[t]{2}{*}{ Ley 617} & $0,112^{*}$ & 0,0502 & 0,0729 \\
\hline & $(0,0485)$ & $(0,0389)$ & $(0,0433)$ \\
\hline \multirow[t]{2}{*}{ Deuda $(t-1)$} & $0,0223^{*}$ & $0,0217^{* *}$ & $0,0221^{*}$ \\
\hline & $(0,00977)$ & $(0,00784)$ & $(0,00873)$ \\
\hline \multirow[t]{2}{*}{ Población } & $1,013^{* *}$ & $0,682^{*}$ & $1,171^{* * *}$ \\
\hline & $(0,332)$ & $(0,268)$ & $(0,298)$ \\
\hline \multirow[t]{2}{*}{ Empresas } & $0,356^{* * *}$ & $0,251^{* * *}$ & $0,296^{* * *}$ \\
\hline & $(0,0422)$ & $(0,0339)$ & $(0,0377)$ \\
\hline \multirow[t]{2}{*}{ Empleo } & $0,0592^{*}$ & $0,0434^{*}$ & 0,0411 \\
\hline & $(0,0259)$ & $(0,0208)$ & $(0,0231)$ \\
\hline \multirow[t]{2}{*}{ Categoría 2} & $-0,0955$ & $-0,119$ & $-0,105$ \\
\hline & $(0,225)$ & $(0,181)$ & $(0,201)$ \\
\hline \multirow[t]{2}{*}{ Categoría 3} & $-0,101$ & $-0,298$ & $-0,0558$ \\
\hline & $(0,253)$ & $(0,203)$ & $(0,226)$ \\
\hline \multirow[t]{2}{*}{ Categoría 4} & $-0,357$ & $-0,573^{*}$ & $-0,511$ \\
\hline & $(0,298)$ & $(0,239)$ & $(0,266)$ \\
\hline \multirow[t]{2}{*}{ Categoría 5} & $-0,347$ & $-0,593^{*}$ & $-0,409$ \\
\hline & $(0,312)$ & $(0,250)$ & $(0,278)$ \\
\hline \multirow[t]{2}{*}{ Categoría 6} & $-0,630^{*}$ & $-0,757^{* *}$ & $-0,584^{*}$ \\
\hline & $(0,320)$ & $(0,256)$ & $(0,286)$ \\
\hline \multirow[t]{2}{*}{ Categoría Especial } & 0,121 & 0,208 & 0,191 \\
\hline & $(0,333)$ & $(0,267)$ & $(0,297)$ \\
\hline Elecciones 2 & $0,755^{* * *}$ & $0,707^{* * *}$ & $0,855^{* * *}$ \\
\hline
\end{tabular}


Tabla 1A. Continuación

\begin{tabular}{lccc}
\hline & $(1)$ & $(2)$ & $(3)$ \\
\hline \multirow{2}{*}{ Elecciones 3 } & $(0,0339)$ & $(0,0272)$ & $(0,0303)$ \\
& $0,362^{* * *}$ & $0,313^{* * *}$ & $0,509^{* * *}$ \\
Elecciones 4 & $(0,0381)$ & $(0,0306)$ & $(0,0341)$ \\
& $0,0979^{*}$ & $-0,00917$ & $0,241^{* * *}$ \\
Constante & $(0,0390)$ & $(0,0313)$ & $(0,0348)$ \\
& $9,205^{* *}$ & $13,08^{* * *}$ & $9,525^{* *}$ \\
\hline$N$ & $(3,322)$ & $(2,676)$ & $(2,974)$ \\
$R^{2}$ & 1.125 & 1.124 & 1.124 \\
\hline
\end{tabular}

Nota: ${ }^{* * *},{ }^{* *},{ }^{*}$ indican niveles de significancia al $1 \%, 5 \%, 10 \%$, respectivamente. Los errores estándar están entre paréntesis.

Fuente: elaboración propia.

\section{Referencias}

Barro, R. J (1990). Government Spending in a Simple Model of Endogenous Growth. Journal of Political Economy, 98(5), 103-125.

Bergman, U. M., Hutchison, M. M. \& Hougaard Jensen, S. E. (2016). Promoting sustainable public finances in the European Union: The role of fiscal rules and government efficiency. European Journal of Political Economy, 44, 1-19.

Camagni, R. (2015). Economía Urbana. Barcelona, España: Antoni Bosch.

Chamorro Narváez, R. A. \& Urrea Bermúdez, A. F. (2016). Incidencia de las reglas fiscales en la sostenibilidad de la deuda pública territorial en colombia. Cuadernos de Economía, 35(67), 207-250.

Faguet, J. P. (2008). Decentralisation's Effects on Public Investment: Evidence and Policy Lessons from Bolivia and Colombia. The Journal of Development Studies, 44(8), 1100-1121. 
Gyourko, J. \& Tracy, J. (2002). The Structure of Local Public Finance and the Quality of Life. Journal of Political Economy, 99(4), 774-806.

Hausman, A. J. \& Taylor, W. E. (1981). Panel Data and Unobservable Individual Effects. Econometrica, 49(6), 1377-1398.

Hausman, J. A. (1978). Specification Tests in Econometrics. Econometrica, $46(6), 1251-1271$.

Lozano, I. \& Julio, J. (2016). Fiscal decentralization and economic growth in Colombia: evidence from regional-level panel data. CEPAL Review, 119, 65-82.

Oates, W. E. (1999). An Essay on Fiscal Federalism. Journal of Economic Literature, 37(3), 1120-1149.

Quintero V., J. F., Gómez N., E. J. \& Guisao, V. D. (2016). Finanzas públicas y calidad de vida: un estudio comparativo para las subregiones Oriente y Suroeste del departamento de Antioquia en el periodo 2004-2014 (Documento de Trabajo, Universidad Autónoma Latinoamericana).

Romero, D. \& Strauch, R. (2008). Públic Finances and long term growth in Europe: Evidence from a panel data analysis. European Journal of Political Economy, 24(1), 172-191.

Thompson, P. N. (2017). Effects of fiscal stress labels on municipal government finances, housing prices, and the quality of public services: Evidence from Ohio. Regional Science and Urban Economics, 64, 98-116.

Tiebout, C. M. (1956). A Pure Theory of Local Expenditure. Journal of Political Economy, 64(5), 416-424.

Tiebout, C. M. (1961). An Economic Theory of Fiscal Descentralization. En Needs, Sources, and Utilization (pp. 79-96). Nueva Jersey: Princeton University Press. 